\title{
FACTORIZATION INVARIANTS OF THE ADDITIVE STRUCTURE OF EXPONENTIAL PUISEUX SEMIRINGS
}

\author{
HAROLD POLO
}

\begin{abstract}
Exponential Puiseux semirings are additive submonoids of $\mathbb{Q}_{\geq 0}$ generated by almost all of the nonnegative powers of a positive rational number, and they are natural generalizations of rational cyclic semirings. In this paper, we investigate some of the factorization invariants of exponential Puiseux semirings and briefly explore the connections of these properties with semigroup-theoretical invariants. Specifically, we provide exact formulas to compute the catenary degrees of these monoids and show that minima and maxima of their sets of distances are always attained at Betti elements. Additionally, we prove that sets of lengths of atomic exponential Puiseux semirings are almost arithmetic progressions with a common bound, while unions of sets of lengths are arithmetic progressions. We conclude by providing various characterizations of the atomic exponential Puiseux semirings with finite omega functions; in particular, we completely describe them in terms of their presentations.
\end{abstract}

\section{INTRODUCTION}

In a unique factorization domain (or UFD) we can write a nonzero nonunit element as a product of finitely many atoms (i.e., irreducibles) and such a representation is unique up to order and units. By relaxing this property, we obtain two larger classes of integral domains that we call atomic and half-factorial following Cohn [15] and Zaks [45], respectively. An integral domain is atomic provided that every nonzero nonunit factors into atoms, while an atomic domain is half-factorial (or HFD) if any two factorizations of a nonunit element have the same length. Factorization theory studies how far is an atomic domain from being either a UFD or an HFD, and several invariants have been introduced to quantify this deviation (see, for example, $[14,22,29,44])$. In 1992, Halter-Koch [40] expanded the scope of factorization theory to the more general (and still suitable) class of cancellative and commutative monoids.

Puiseux monoids (i.e., additive submonoids of $\mathbb{Q}_{>0}$ ) are natural generalizations of numerical monoids, and they have been used as an important source of examples in factorization theory. For instance, Coykendall and Gotti [17] utilized Puiseux monoids to partially answer a question posed by Gilmer almost forty years ago in [34, page 189],

Date: December 2, 2021.

2010 Mathematics Subject Classification. Primary: 20M13; Secondary: 16Y60, 06F05, $20 \mathrm{M} 14$.

Key words and phrases. exponential Puiseux semirings, rational cyclic semirings, sets of lengths, sets of distances, catenary degrees, omega primalities, tame degrees, Betti elements, presentations. 
H. POLO

while Geroldinger et al. [24] found, in the class of Puiseux monoids, the first example of an atomic primary monoid with irrational elasticity ( [24, Example 4.3]). Computations of factorization invariants are highly tractable in the context of finitely generated monoids (see [20] and references therein); however, computations are usually arduous for their non-finitely generated counterparts. For example, completely describing the sets of lengths of certain Puiseux monoids is as hard as solving the Goldbach's conjecture ([36, Section 6]).

Some additive submonoids of $\mathbb{R}_{\geq 0}$ have dyadic monoidal structures in the sense that they contain 1, the multiplicative identity, and are also closed under multiplication. These monoids, called positive semirings, have motivated much interest lately. In [16], the authors studied atomic properties of the additive structure of the positive semiring $\mathbb{N}_{0}[\alpha]=\left\{f(\alpha) \mid f(X) \in \mathbb{N}_{0}[X]\right\}$, where $\alpha$ is a nonzero real number, while the elasticity and delta set of $\mathbb{N}_{0}[\tau]$, where $\tau$ is a quadratic integer, were explored in [8]. Furthermore, Baeth et al. [4] investigated the dual nature of atomicity in the context of positive semirings using a methodology introduced in [2]. Finally, the factorization invariants of the additive structure of rational cyclic semirings (i.e., Puiseux monoids generated by the nonnegative powers of a positive rational number) were thoroughly described in [12], while the arithmetic of their multiplicative structure was considered in [5].

The class of atomic rational cyclic semirings is one of the rare classes of non-finitely generated monoids for which we can provide detailed descriptions for many of their factorization invariants, and various generalizations of this class (e.g., [1,16,43]) have been scrutinized to gain insight about the atomic structure and factorization invariants of positive monoids (i.e., additive submonoids of the nonnegative cone of the real line). In [1], Albizu-Campos et al. studied the atomic properties of exponential Puiseux semirings, that is, Puiseux monoids generated by almost all of the nonnegative powers of a positive rational number. The purpose of the present article is to show that atomic exponential Puiseux semirings retain some of the nice factorization properties of atomic rational cyclic semirings.

We start by introducing the necessary background and notation to follow our exposition. In Section 3, we describe the $\mathcal{R}$-classes of atomic exponential Puiseux semirings, which allows us to compute their catenary degrees. Additionally, we show that the minimum and maximum of $\Delta(M)$ are attained at Betti elements for every atomic exponential Puiseux semiring $M$. Then, in Section 4, we investigate the sets of lengths of these monoids. To be precise, we prove that if $M$ is a nontrivial atomic exponential Puiseux semiring then there exists $B \in \mathbb{N}$ such that every $L \in \mathcal{L}(M)$ is an AAP with difference $\min \Delta(M)$ and bound $B$, while for each $k \in \mathbb{N}^{\bullet}$ the set $\mathcal{U}_{k}(M)$ is an arithmetic sequence with difference $\min \Delta(M)$. We conclude by offering, in the last section, several characterizations of atomic exponential Puiseux semirings with finite omega functions; in particular, we completely describe them in terms of their presentations. 


\section{Fundamentals}

We now present the concepts and notation related to our exposition. Reference material on non-unique factorization theory can be found in the monograph [26] by Geroldinger and Halter-Koch.

2.1. Notation. Let $\mathbb{N}$ denote the set of nonnegative integers, and let $\mathbb{P}$ denote the set of prime numbers. Additionally, if $X$ is a subset of the rational numbers then we set $\bar{X}:=X \cup\{\infty\}$ and $X_{<q}:=\{x \in X \mid 0 \leq x<q\}$; we define $X_{\leq q}, X_{>q}$, and $X_{\geq q}$ in a similar way. For a positive rational number $r=n / d$ with $n$ and $d$ relatively prime positive integers, we call $n$ the numerator and $d$ the denominator of $r$, and we set $\mathrm{n}(r):=n$ and $\mathrm{d}(r):=d$. For nonnegative integers $k$ and $m$, we denote by $\llbracket k, m \rrbracket$ the set of integers between $k$ and $m$, i.e.,

$$
\llbracket k, m \rrbracket:=\{s \in \mathbb{N} \mid k \leq s \leq m\} .
$$

Given $L, L_{1}, \ldots, L_{n} \subseteq \mathbb{Z}$, we denote by $L_{1}+\cdots+L_{n}$ the set $\left\{l_{1}+\cdots+l_{n} \mid l_{i} \in L_{i}\right\}$ and, for $l \in \mathbb{Z}$, we set $l+L:=\{l\}+L$.

2.2. Exponential Puiseux Semirings. Unless we specify otherwise, a monoid is defined to be a semigroup with identity that is cancellative, commutative, and reduced (i.e., its only invertible element is the identity) and, throughout this paper, we use additive notation for monoids. Now let $M$ be a monoid. We denote by $\mathcal{A}(M)$ the set consisting of elements $a \in M^{\bullet}:=M \backslash\{0\}$ satisfying that if $a=x+y$ for some $x, y \in M$ then either $x=0$ or $y=0$; the elements of this set are called atoms. For a subset $G \subseteq M$, we denote by $\langle G\rangle$ the smallest submonoid of $M$ containing $G$, and if $M=\langle G\rangle$ then it is said that $G$ is a generating set of $M$. A monoid $M$ is atomic if $M=\langle\mathcal{A}(M)\rangle$. For $x, y \in M$, it is said that $x$ divides $y$ if there exists $x^{\prime} \in M$ such that $y=x+x^{\prime}$ in which case we write $\left.x\right|_{M} y$ and drop the subscript whenever $M=(\mathbb{N}, \times)$. A subset $I$ of $M$ is an ideal of $M$ provided that $I+M \subseteq I$. An ideal $I$ is principal if $I=x+M$ for some $x \in M$. Furthermore, it is said that $M$ satisfies the ascending chain condition on principal ideals (or $A C C P$ ) if every increasing sequence of principal ideals of $M$ eventually stabilizes.

A numerical monoid $S$ is an additive submonoid of $\mathbb{N}$ whose complement in $\mathbb{N}$ is finite; the greatest integer that is not an element of $S$ is called the Frobenius number of $S$ and is denoted by $F(S)$. It is well known that numerical monoids are always finitely generated and, therefore, atomic. An introduction to numerical monoids can be found in [21]. On the other hand, Puiseux monoids are additive submonoids of $\mathbb{Q}_{\geq 0}$, so they are natural generalizations of numerical monoids. The factorization invariants of these monoids have received considerable attention during the past five years (see, for instance, $[36,38,39])$. A particularly captivating class of Puiseux monoids is the one consisting of monoids generated by the nonnegative powers of a positive rational number. These monoids, called rational cyclic semirings, were first considered in [37], 
H. POLO

and a detailed description of their factorization invariants was provided in [12]. See [13] for a friendly survey about Puiseux monoids.

Definition 2.1. Take $r \in \mathbb{Q}_{>0}$ and let $S$ be a numerical monoid. We let $M_{r, S}$ denote the monoid

$$
M_{r, S}:=\left\langle r^{k} \mid k \in S\right\rangle
$$

which we call exponential Puiseux semiring. For $n \in \mathbb{N}$, we denote by $s_{n}$ the $(n+1)$ th smallest element of $S$ and set $\delta_{n}:=s_{n+1}-s_{n}$.

With notation as in Definition 2.1, if $r$ is a positive integer then we say that $M_{r, S}$ (which is just $\mathbb{N}$ ) is the trivial exponential Puiseux semiring. By virtue of [1, Proposition 5.1], the monoid $M_{r, S}$ is, in fact, a positive semiring (i.e., an additive submonoid of the real line containing 1 and closed under multiplication). Clearly, exponential Puiseux semirings are generalizations of rational cyclic semirings, and most of them are atomic as the next proposition indicates.

Proposition 2.2. [1, Proposition 3.7] Let $M_{r, S}$ be a nontrivial exponential Puiseux semiring. The following statements hold.

(1) If $\mathrm{n}(r)>1$ and $\mathrm{d}(r)>1$ then $M_{r, S}$ is atomic and $\mathcal{A}\left(M_{r, S}\right)=\left\{r^{s} \mid s \in S\right\}$.

(2) If $\mathrm{n}(r)=1$ and $\mathrm{d}(r)>1$ then $M_{r, S}$ is not atomic and $\mathcal{A}\left(M_{r, S}\right)=\emptyset$.

2.3. Factorizations. For the rest of the section, let $M$ be an atomic monoid. The factorization monoid of $M$, denoted by $\mathbf{Z}(M)$, is the free (commutative) monoid on $\mathcal{A}(M)$. The elements of $\mathrm{Z}(M)$ are called factorizations, and if $z=a_{1}+\cdots+a_{n} \in \mathbf{Z}(M)$ for $a_{1}, \ldots, a_{n} \in \mathcal{A}(M)$ then it is said that the length of $z$, denoted by $|z|$, is $n$. We assume that the empty factorization has length 0 . The unique monoid homomorphism $\pi: \mathrm{Z}(M) \rightarrow M$ satisfying that $\pi(a)=a$ for all $a \in \mathcal{A}(M)$ is called the factorization homomorphism of $M$. For each $x \in M$, there are two important sets associated to $x$ :

$$
\mathrm{Z}_{M}(x):=\pi^{-1}(x) \subseteq \mathrm{Z}(M) \quad \text { and } \quad \mathrm{L}_{M}(x):=\left\{|z|: z \in \mathrm{Z}_{M}(x)\right\}
$$

which are called the set of factorizations of $x$ and the set of lengths of $x$, respectively; we drop the subscript whenever the monoid is clear from the context. Note that $\mathrm{L}(0)=\{0\}$. Additionally, the system of sets of lengths of $M$ is defined by

$$
\mathcal{L}(M):=\{\mathrm{L}(x) \mid x \in M\} .
$$

See [23] for a survey on sets of lengths. It is said that $M$ is a finite factorization monoid (or an FFM) if $\mathrm{Z}(x)$ is nonempty and finite for all $x \in M$. Similarly, $M$ is a bounded factorization monoid (or BFM) if $\mathrm{L}(x)$ is nonempty and finite for all $x \in M$. We say that an element $x \in M$ divides a factorization $z \in \mathbf{Z}(M)$ provided that $x$ divides $\pi(z)$ in $M$. 
2.4. Presentations and Betti Elements. Let $\sigma$ be a subset of $M \times M$. Then we set

$$
\sigma^{t}:=\{(s+x, r+x) \mid(s, r) \in \sigma \text { and } x \in M\} .
$$

On the other hand, we let $\sigma^{e}$ denote the smallest (under inclusion) equivalence relation on $M$ containing $\sigma$. The relation $\sigma$ on $M$ is called a congruence provided that $\sigma$ is an equivalence relation satisfying that $\sigma^{t}=\sigma$. The congruence generated by $\sigma$, denoted by $\sigma^{\sharp}$, is the smallest (under inclusion) congruence on $M$ containing $\sigma$.

Proposition 2.3. [41, Proposition 1.5.9] If $M$ is a monoid and $\rho \subseteq M \times M$ then $\rho^{\sharp}=\left(\rho^{t}\right)^{e}$.

Let $\psi: M \rightarrow M^{\prime}$ be a monoid homomorphism. Then $\operatorname{ker} \psi:=\{(a, b) \in M \times M \mid$ $\psi(a)=\psi(b)\}$ is called the kernel congruence of $M$ with respect to $\psi$. The kernel congruence of the factorization homomorphism $\pi: \mathrm{Z}(M) \rightarrow M$ is denoted by $\sim_{M}$. If $\rho \subseteq \mathrm{Z}(M) \times \mathbf{Z}(M)$ generates $\sim_{M}$ in the sense that $\sim_{M}$ is the smallest (under inclusion) congruence of $\mathbf{Z}(M)$ containing $\rho$ then it is said that $\rho$ is a presentation of $M$.

Given two factorizations $z=\sum_{a \in \mathcal{A}(M)} \alpha_{a} a$ and $z^{\prime}=\sum_{a \in \mathcal{A}(M)} \beta_{a} a$ in Z $(x)$, we set $\operatorname{gcd}\left(z, z^{\prime}\right):=\sum_{a \in \mathcal{A}(M)} \min \left(\alpha_{a}, \beta_{a}\right) a$ and $\mathrm{d}\left(z, z^{\prime}\right):=\max \left\{|z|,\left|z^{\prime}\right|\right\}-\left|\operatorname{gcd}\left(z, z^{\prime}\right)\right|$. For $x \in M$ and $z, z^{\prime} \in \mathbf{Z}(x)$, a connecting chain of factorizations or, simply, a connecting chain from $z$ to $z^{\prime}$ is an ordered chain of factorizations $z=z_{1}, \ldots, z_{n}=z^{\prime} \in \mathrm{Z}(x)$ such that $\operatorname{gcd}\left(z_{i}, z_{i+1}\right) \in \mathbf{Z}(M)^{\bullet}$ for each $i \in \llbracket 1, n-1 \rrbracket$. Now consider the binary relation $\mathcal{R}$ on $\mathrm{Z}(M)$ defined as follows: $\left(z, z^{\prime}\right) \in \mathcal{R}$ if there exists a connecting chain from $z$ to $z^{\prime}$. Evidently, $\mathcal{R} \subseteq \operatorname{ker} \pi$. Given $x \in M$, we denote by $\mathcal{R}_{x}$ the set consisting of all $\mathcal{R}$-classes of $\mathrm{Z}(x)$, and $x$ is called a Betti element of $M$ provided that $\left|\mathcal{R}_{x}\right|>1$. The set of Betti elements of $M$ is denoted by $\operatorname{Betti}(M)$.

\section{Betti Elements, Catenary Degrees, and Sets of Distances}

Several papers have been devoted to study the catenary degree (see definition below) in atomic monoids (e.g., [9, 11, 25,32]). However, exact formulas to calculate this invariant are hard to come by, even in the context of finitely generated monoids.

Definition 3.1. Let $M$ be an atomic monoid, and let $x$ be an element of $M$.

(1) For $n \in \overline{\mathbb{N}}$, a finite sequence $z_{1}, \ldots, z_{k} \in \mathbf{Z}(x)$ is called an $n$-chain of factorizations connecting $z_{1}$ with $z_{k}$ if $\mathrm{d}\left(z_{i}, z_{i+1}\right) \leq n$ for each $i \in \llbracket 1, k-1 \rrbracket$.

(2) The catenary degree of $x$, denoted by $\mathrm{c}(x)$, is the smallest $n \in \overline{\mathbb{N}}$ for which any two factorizations in $\mathrm{Z}(x)$ are connected through an $n$-chain.

(3) The catenary degree of $M$, denoted by $\mathrm{c}(M)$, is defined by $\mathrm{c}(M):=\sup \{\mathrm{c}(x) \mid$ $x \in M\}$.

In this section, we describe the $\mathcal{R}$-classes of nontrivial atomic exponential Puiseux semirings. This will allow us to compute their catenary degrees via [42, Corollary 9], an approach considerable different than that adopted in [12, Corollary 3.4] to compute 
H. POLO

the catenary degrees of atomic rational cyclic semirings. But first let us collect two technical lemmas; the first one was borrowed from [1] (Lemma 3.8), and the second one is its counterpart for the case where $r>1$.

Lemma 3.2. [1, Lemma 3.8] Let $x$ be a nonzero element of an atomic exponential Puiseux semiring $M_{r, S}$ with $r \in \mathbb{Q}_{<1}$, and consider a factorization $z=\sum_{i=0}^{n} c_{i} r^{s_{i}} \in$ $\mathrm{Z}(x)$ with coefficients $c_{0}, \ldots, c_{n} \in \mathbb{N}$. The following conditions hold.

(1) $\min \mathrm{L}(x)=|z|$ if and only if $c_{i}<\mathrm{d}(r)^{\delta_{i-1}}$ for each $i \in \llbracket 1, n \rrbracket$.

(2) There exists exactly one factorization $z_{\min } \in \mathrm{Z}(x)$ of minimum length.

(3) $\max \mathrm{L}(x)=|z|$ if and only if $c_{i}<\mathrm{n}(r)^{\delta_{i}}$ for each $i \in \llbracket 0, n \rrbracket$.

(4) There exists, at most, one factorization $z_{\max } \in \mathbf{Z}(x)$ of maximum length.

(5) If $c_{i}<\mathrm{n}(r)$ for every $i \in \llbracket 0, n \rrbracket$ then $|\mathrm{Z}(x)|=1$.

Lemma 3.3. Let $x$ be a nonzero element of an atomic exponential Puiseux semiring $M_{r, S}$ with $r \in \mathbb{Q}_{>1} \backslash \mathbb{N}$, and consider a factorization $z=\sum_{i=0}^{n} c_{i} r^{s_{i}} \in \mathbf{Z}(x)$ with coefficients $c_{0}, \ldots, c_{n} \in \mathbb{N}$. The following conditions hold.

(1) $\min \mathrm{L}(x)=|z|$ if and only if $c_{i}<\mathrm{n}(r)^{\delta_{i}}$ for each $i \in \llbracket 0, n \rrbracket$.

(2) There exists exactly one factorization $z_{\min } \in \mathbf{Z}(x)$ of minimum length.

(3) $\max \mathrm{L}(x)=|z|$ if and only if $c_{i}<\mathrm{d}(r)^{\delta_{i-1}}$ for each $i \in \llbracket 1, n \rrbracket$.

(4) There exists exactly one factorization $z_{\max } \in \mathbf{Z}(x)$ of maximum length.

(5) If $c_{i}<\mathrm{d}(r)$ for every $i \in \llbracket 0, n \rrbracket$ then $|\mathrm{Z}(x)|=1$.

Proof. The proofs of (1), (2), (3), and (4) are left to the reader as they mimick the proof of the corresponding parts of [12, Lemma 3.2]. And (5) readily follows from (1) and (3).

For the rest of the paper we assume, without explicitly mentioning, that given an element $x$ in an atomic exponential Puiseux semiring $M_{r, S}$, the set $\mathbf{Z}(x)$ contains exactly one factorization of minimum length; we also assume that $\mathrm{Z}(x)$ contains exactly one factorization of maximum length provided that $r>1$.

Remark 3.4. Let $M_{r, S}$ be a nontrivial atomic exponential Puiseux semiring, and let $x$ be an element of $M_{r, S}$. Given $z \in \mathrm{Z}(x)$, there exist factorizations $z=z_{1}, \ldots, z_{k}=$ $z_{\text {min }} \in \mathrm{Z}(x)$ such that $z_{\text {min }}$ is the factorization of minimum length of $x$ and, for each $i \in \llbracket 1, k-1 \rrbracket$, we have $\left|z_{i}\right|-\left|z_{i+1}\right|=\left|\mathrm{n}(r)^{\delta_{m}}-\mathrm{d}(r)^{\delta_{m}}\right|$ for some $m \in \mathbb{N}$. We can generate such a sequence of factorizations iteratively: set $z_{1}:=z$, and assume that we already defined $z_{m}=\sum_{i=0}^{n} c_{i} r^{s_{i}} \in \mathbf{Z}(x)$ for some $m \in \mathbb{N}^{\bullet}$. If $z_{m}$ is not the factorization of minimum length of $x$ and $r<1$ (resp. $r>1$ ) then $c_{j} \geq \mathrm{d}(r)^{\delta_{j-1}}\left(\operatorname{resp} . c_{j} \geq \mathrm{n}(r)^{\delta_{j}}\right.$ ) for some $j \in \llbracket 1, n \rrbracket($ resp. $j \in \llbracket 0, n \rrbracket)$ by Lemma 3.2 (resp. Lemma 3.3). By applying the transformation

$$
\mathrm{d}(r)^{\delta_{j-1}} r^{s_{j}}=\mathrm{n}(r)^{\delta_{j-1}} r^{s_{j-1}}\left(\text { resp. } \mathrm{n}(r)^{\delta_{j}} r^{s_{j}}=\mathrm{d}(r)^{\delta_{j}} r^{s_{j+1}}\right)
$$

we can generate a factorization $z_{m+1} \in \mathrm{Z}(x)$ satisfying $\left|z_{m}\right|-\left|z_{m+1}\right|=\left|\mathrm{n}(r)^{\delta_{t}}-\mathrm{d}(r)^{\delta_{t}}\right|$ for some $t \in \mathbb{N}$. Consequently, the inequality $\left|z_{m}\right|>\left|z_{m+1}\right|$ holds which, in turn, 
implies that these iterations eventually stop. By Lemma 3.2 (resp. Lemma 3.3), the last factorization we obtained is precisely the factorization of minimum length of $x$. A similar statement is true, mutatis mutandis, if we take $r>1$ and $z_{k} \in \mathbf{Z}(x)$ to be the factorization of maximum length of $x$.

The following example illustrates how to go from one factorization to another of the same element using the transformations described in Remark 3.4.

Example 3.5. Let $r=2 / 3$ and $S=\{0,5,6,8,9\} \cup \mathbb{N}_{\geq 11}$, and consider the Puiseux semiring $M_{r, S}$. Clearly, $z=3(2 / 3)^{6}+2(2 / 3)^{8}, z_{\min }=2(2 / 3)^{5}+2(2 / 3)^{8}$, and $z^{\prime}=$ $2(2 / 3)^{5}+3(2 / 3)^{9}$ are factorizations of the same element $x \in M_{r, S}$. Note that $z_{\text {min }}$ is, in fact, the factorization of minimum length of $x$. By performing the transformations $3(2 / 3)^{6}=2(2 / 3)^{5}$ and $2(2 / 3)^{8}=3(2 / 3)^{9}$, we can go from the factorization $z$ to $z_{\min }$ and from $z_{\min }$ to $z^{\prime}$, respectively.

We are now in a position to describe the $\mathcal{R}$-classes of nontrivial atomic exponential Puiseux semirings.

Proposition 3.6. Let $M_{r, S}$ be a nontrivial atomic exponential Puiseux semiring. Let $x \in M_{r, S}$, and set $R_{x}\left(r^{s_{m}}\right):=\mathrm{Z}(x) \cap\left(r^{s_{m}}+\mathrm{Z}\left(M_{r, S}\right)\right)$ for $m \in \mathbb{N}$. The following statements hold.

(1) $\operatorname{Betti}\left(M_{r, S}\right)=\left\{\mathrm{n}(r)^{\delta_{n}} r^{s_{n}} \mid n \in \mathbb{N}\right\}$.

(2) If $x=\mathrm{n}(r)^{\delta_{n}} r^{s_{n}}$ for some $n \in \mathbb{N}$ then $\mathcal{R}_{x}=\left\{R_{x}\left(r^{s_{n}}\right), R_{x}\left(r^{s_{n+1}}\right)\right\}$.

Proof. Fix $n \in \mathbb{N}$, and take $x=\mathrm{n}(r)^{\delta_{n}} r^{s_{n}} \in M_{r, S}$. Let $A=\left\{r^{s_{0}}, \ldots, r^{s_{n}}\right\}$ and $B=$ $\mathcal{A}\left(M_{r, S}\right) \backslash A$. Consider the factorizations $z=\mathrm{n}(r)^{\delta_{n}} r^{s_{n}}$ and $z^{\prime}=\mathrm{d}(r)^{\delta_{n}} r^{s_{n+1}}$ in Z $(x)$. By Remark 3.4, if $r<1$ (resp. $r>1$ ) then the factorization of minimum (resp. maximum) length of $x$ does not contain atoms from the subset $B$. By way of contradiction, suppose that there exists a factorization $z^{\prime \prime} \in \mathrm{Z}(x)$ containing atoms from both subsets $A$ and $B$. If $r<1$ (resp. $r>1$ ) then by repeatedly applying the identity $\mathrm{d}(r)^{\delta_{m}} r^{s_{m+1}}=\mathrm{n}(r)^{\delta_{m}} r^{s_{m}}$ we can generate factorizations $z^{\prime \prime}=z_{1}, \ldots, z_{k} \in \mathrm{Z}(x)$ such that $z_{k}$ is the factorization of minimum (resp. maximum) length of $x$ and, for each $i \in \llbracket 1, k-1 \rrbracket$, we have $\left|z_{i}\right|-\left|z_{i+1}\right|=\left|\mathrm{n}(r)^{\delta_{m}}-\mathrm{d}(r)^{\delta_{m}}\right|$ (resp. $\left.\left|z_{i+1}\right|-\left|z_{i}\right|=\left|\mathrm{n}(r)^{\delta_{m}}-\mathrm{d}(r)^{\delta_{m}}\right|\right)$ for some $m \in \mathbb{N}$. But since $z^{\prime \prime}$ contains atoms from $B$ and $z_{k}$ does not, at some point in generating $z_{1}, \ldots, z_{k}$ we applied the transformation $\mathrm{d}(r)^{\delta_{n}} r^{s_{n+1}}=\mathrm{n}(r)^{\delta_{n}} r^{s_{n}}$, which is a contradiction given that $z^{\prime \prime}$ also contains atoms from $A$. Hence $z$ and $z^{\prime}$ belong to different $\mathcal{R}$-classes of $\mathrm{Z}(x)$, from which the inclusion $\left\{\mathrm{n}(r)^{\delta_{n}} r^{s_{n}} \mid n \in \mathbb{N}\right\} \subseteq \operatorname{Betti}\left(M_{r, S}\right)$ follows.

Note that if the atoms ocurring in a factorization $z^{*} \in \mathrm{Z}(x)$ are in $A$ then, by a similar argument to that one used in Remark 3.4, there exist factorizations $z=$ $z_{1}^{*}, \ldots, z_{t}^{*}=z^{*} \in \mathrm{Z}(x)$ such that, for each $i \in \llbracket 2, t \rrbracket$, one can obtain $z_{i}^{*}$ from $z_{i-1}^{*}$ by performing a transformation of the form $\mathrm{d}(r)^{\delta_{m}} r^{s_{m+1}}=\mathrm{n}(r)^{\delta_{m}} r^{s_{m}}$ for some $m \in \mathbb{N}$. Since $\mathrm{d}(r)^{\delta_{k-1}} \nmid \mathrm{n}(r)^{\delta_{k}}$ for any $k \in \mathbb{N}^{\bullet}$, the atom $r^{s_{n}}$ shows up in the factorization $z_{i}^{*}$ 
H. POLO

for each $i \in \llbracket 1, t \rrbracket$. Consequently, all factorizations of $x$ with atoms in $A$ are in the same $\mathcal{R}$-class, namely $R_{x}\left(r^{s_{n}}\right)$. Similarly, all factorizations of $x$ with atoms in $B$ are in $R_{x}\left(r^{s_{n+1}}\right)$, from which (2) follows.

To verify that the inclusion $\operatorname{Betti}\left(M_{r, S}\right) \subseteq\left\{\mathrm{n}(r)^{\delta_{n}} r^{s_{n}} \mid n \in \mathbb{N}\right\}$ holds, let $x \in$ $M_{r, S} \backslash\left\{\mathrm{n}(r)^{\delta_{n}} r^{s_{n}} \mid n \in \mathbb{N}\right\}$, and let $z_{\min } \in \mathrm{Z}(x)$ be the factorization of minimum length of $x$. For every $z \in \mathbf{Z}(x)$, there exist factorizations $z=z_{1}, \ldots, z_{k}=z_{\min } \in \mathbf{Z}(x)$ such that, for each $i \in \llbracket 1, k-1 \rrbracket$, we have $\left|z_{i}\right|-\left|z_{i+1}\right|=\left|\mathrm{n}(r)^{\delta_{m}}-\mathrm{d}(r)^{\delta_{m}}\right|$ for some $m \in \mathbb{N}$ by Remark 3.4; since we generate such a sequence of factorizations through transformations of the form $\mathrm{n}(r)^{\delta_{k}} r^{s_{k}}=\mathrm{d}(r)^{\delta_{k}} r^{s_{k+1}}$, we have $\operatorname{gcd}\left(z_{i}, z_{i+1}\right) \neq 0$ unless $z_{i}=\mathrm{n}(r)^{\delta_{j}} r^{s_{j}}$ or $z_{i+1}=\mathrm{n}(r)^{\delta_{j}} r^{s_{j}}$ for some $j \in \mathbb{N}$, but this is impossible since $x \notin$ $\left\{\mathrm{n}(r)^{\delta_{n}} r^{s_{n}} \mid n \in \mathbb{N}\right\}$. Consequently, there is a chain connecting $z$ and $z_{\text {min }}$ which, in turn, implies $\left|\mathcal{R}_{x}\right|=1$, and our proof concludes.

Corollary 3.7. Let $M_{r, S}$ be a nontrivial atomic exponential Puiseux semiring. Then

$$
\mathrm{c}\left(M_{r, S}\right)=\max (\mathrm{n}(r), \mathrm{d}(r))^{\delta_{0}} .
$$

Corollary 3.8. Let $M_{r, \mathbb{N}}$ be a nontrivial atomic rational cyclic semiring. Then

$$
\mathrm{c}\left(M_{r, \mathbb{N}}\right)=\max (\mathrm{n}(r), \mathrm{d}(r)) .
$$

For the rest of the section, we focus on the factorization invariant known as set of distances or delta set. This invariant has been extensively studied in the context of BFMs, including numerical monoids [6] and transfer Krull monoids [33].

Definition 3.9. Let $M$ be an atomic monoid, and let $x$ be an element of $M$.

(1) It is said that $d \in \mathbb{N} \bullet$ is a distance of $x$ provided that $\mathrm{L}(x) \cap \llbracket l, l+d \rrbracket=\{l, l+d\}$ for some $l \in \mathrm{L}(x)$.

(2) The set of distances of $x$, denoted by $\Delta(x)$, is the set consisting of all the distances of $x$.

(3) The set $\Delta(M):=\bigcup_{x \in M} \Delta(x)$ is called the set of distances of $M$.

We now show that atomic exponential Puiseux semirings have finite sets of distances. Additionally, we compute the minimum and maximum of $\Delta\left(M_{r, S}\right)$ and proved that they are both attained at Betti elements for all atomic exponential Puiseux semirings $M_{r, S}$.

Proposition 3.10. Let $M_{r, S}$ be a nontrivial atomic exponential Puiseux semiring. Then

$$
\left\{\left|\mathrm{n}(r)^{\delta_{n}}-\mathrm{d}(r)^{\delta_{n}}\right|: n \in \mathbb{N}\right\} \subseteq \Delta\left(M_{r, S}\right) \subseteq \llbracket|\mathrm{n}(r)-\mathrm{d}(r)|,\left|\mathrm{n}(r)^{\delta_{0}}-\mathrm{d}(r)^{\delta_{0}}\right| \rrbracket .
$$

Proof. The first inclusion and the fact that $\Delta\left(M_{r, S}\right)$ is finite follow almost immediately from Remark 3.4. On the other hand, it is easy to mimic the proof of [10, Theorem 2.5] to show that $\max \Delta\left(M_{r, S}\right)$ is achieved at a Betti element. We leave all these details to the reader. Our result follows then from Remark 3.4 and Proposition 3.6. 
Corollary 3.11. Let $M_{r, \mathbb{N}}$ be a nontrivial atomic rational cyclic semiring. Then $\Delta\left(M_{r, \mathbb{N}}\right)=\{|\mathrm{n}(r)-\mathrm{d}(r)|\}$.

The inclusions in (3.1) might be proper. Consider the following example.

Example 3.12. Let $r=2 / 3$, and consider the following numerical monoids

$$
S=\{0,18,19,25,27\} \cup \mathbb{N}_{\geq 36} \quad \text { and } \quad S^{\prime}=\mathbb{N}_{\geq 2} \cup\{0\} .
$$

Clearly, the monoids $M_{r, S}$ and $M_{r, S^{\prime}}$ are both atomic. Moreover, it is not hard to check

$$
\mathrm{L}_{M_{r, S}}\left(2(2 / 3)^{18}+4(2 / 3)^{25}\right)=\{6,7,11,12\},
$$

which implies that $4 \in \Delta\left(M_{r, S}\right)$, but $4 \notin\left\{\left|\mathrm{n}(r)^{\delta_{n}}-\mathrm{d}(r)^{\delta_{n}}\right|: n \in \mathbb{N}\right\}$, where the $\delta_{n}$ 's are taken with respect to the numerical monoid $S$. On the other hand, we have $\Delta\left(M_{r, S^{\prime}}\right)=$ $\{1,5\}$ as the reader can verify, so $\Delta\left(M_{r, S^{\prime}}\right) \subsetneq \llbracket|\mathrm{n}(r)-\mathrm{d}(r)|,\left|\mathrm{n}(r)^{\delta_{0}}-\mathrm{d}(r)^{\delta_{0}}\right| \rrbracket$, where $\delta_{0}=2$.

Proposition 3.13. Let $M_{r, S}$ be a nontrivial atomic exponential Puiseux semiring. Then

$$
\min \Delta\left(M_{r, S}\right)=|\mathrm{n}(r)-\mathrm{d}(r)| \quad \text { and } \quad \max \Delta\left(M_{r, S}\right)=\left|\mathrm{n}(r)^{\delta_{0}}-\mathrm{d}(r)^{\delta_{0}}\right|
$$

are both attained at Betti elements.

Proof. By Proposition 3.10, we have that the equalities min $\Delta\left(M_{r, S}\right)=|\mathrm{n}(r)-\mathrm{d}(r)|$ and $\max \Delta\left(M_{r, S}\right)=\left|\mathrm{n}(r)^{\delta_{0}}-\mathrm{d}(r)^{\delta_{0}}\right|$ hold. Furthermore, we have that $|\mathrm{n}(r)-\mathrm{d}(r)|$ (resp. $\left.\ln (r)^{\delta_{0}}-\mathrm{d}(r)^{\delta_{0}} \mid\right)$ is a distance of $\mathrm{n}(r) r^{F(S)+1}$ (resp. $\left.\mathrm{n}(r)^{\delta_{0}} r^{s_{0}}\right)$. Our argument concludes after noticing that both elements $\mathrm{n}(r) r^{F(S)+1}$ and $\mathrm{n}(r)^{\delta_{0}} r^{s_{0}}$ are Betti elements of $M_{r, S}$ by Proposition 3.6.

In [10], it was proved that, for every bounded factorization monoid $M$, the minimum and maximum of $\Delta(M)$ are both attained at Betti elements. But notice that atomic exponential Puiseux semirings are not, in general, BFMs (see [1, Example 4.7]).

\section{Sets of Lengthis And Their Unions}

For a positive integer $d$ and a nonnegative integer $B$, a subset $L \subseteq \mathbb{Z}$ is called an almost arithmetic progression (or $A A P$ ) with difference $d$ and bound $B$ if

$$
L=y+\left(L^{\prime} \cup L^{*} \cup L^{\prime \prime}\right) \subseteq y+d \mathbb{Z},
$$

where $y \in \mathbb{Z}$ and $L^{*}$ is a nonempty arithmetic progression with difference $d$ such that $\min L^{*}=0, L^{\prime} \subseteq[-B,-1]$, and $L^{\prime \prime} \subseteq \sup L^{*}+[1, B]$. If the set $L^{*}$ has infinite cardinality then we assume that $L^{\prime \prime}=\emptyset$. As the name indicates, almost arithmetic progressions are generalizations of arithmetic sequences, and they have been used in factorization theory to describe sets of lengths of various classes of monoids (see, for instance, [26, Theorem 4.3.6] and [18, Theorem 4.2]). 
In this section, we show that sets of lengths of atomic exponential Puiseux semirings are well structured. Specifically, we prove that if $M_{r, S}$ is a nontrivial atomic exponential Puiseux semiring then there exists $B \in \mathbb{N}$ such that every $L \in \mathcal{L}\left(M_{r, S}\right)$ is an AAP with difference $|\mathrm{n}(r)-\mathrm{d}(r)|$ and bound $B$.

Theorem 4.1. Let $M_{r, S}$ be a nontrivial atomic exponential Puiseux semiring. There exists $B \in \mathbb{N}$ such that every $L \in \mathcal{L}\left(M_{r, S}\right)$ is an $A A P$ with difference $|\mathrm{n}(r)-\mathrm{d}(r)|$ and bound $B$.

Proof. Clearly, there exists $m \in \mathbb{N}$ such that $s_{m}=F(S)+1$, where $F(S)$ represents the Frobenius number of $S$. Let $x$ be an arbitrary element of $M_{r, S}$, and let $z_{\min }=$ $\sum_{i=0}^{n} c_{i} r^{s_{i}} \in \mathrm{Z}(x)$ with coefficients $c_{0}, \ldots, c_{n} \in \mathbb{N}$ be the factorization of minimum length of $x$. Obviously, we may assume that $m \leq n$. Before continuing with the proof, we introduce a definition. Given a factorization $z=\sum_{i=0}^{k} d_{i} r^{s_{i}}$ with coefficients $d_{0}, \ldots, d_{k} \in \mathbb{N}$, we say that the sub-factorizations $\sum_{i=0}^{m} d_{i} r^{s_{i}}$ and $\sum_{i=m+1}^{k} d_{i} r^{s_{i}}$ are the prefix and suffix of $z$, respectively. We distinguish two cases.

CASE 1: $r<1$. Let $B_{1}=\mathrm{d}(r)^{s_{m}}-\mathrm{n}(r)^{s_{m}}$, and let us denote by $B_{2}$ the maximum number of times we can consecutively apply the identity $\mathrm{n}(r)^{\delta_{\alpha}} r^{s_{\alpha}}=\mathrm{d}(r)^{\delta_{\alpha}} r^{s_{\alpha+1}}$ with $\alpha \in \llbracket 0, m-1 \rrbracket$ to increase the length of the prefix of a factorization $z=\sum_{i=0}^{k} d_{i} r^{s_{i}}$ satisfying that $d_{i}<\mathrm{n}(r)^{s_{m}-s_{i}}$ for each $i \in \llbracket 0, m-1 \rrbracket$. Evidently, neither $B_{1}$ nor $B_{2}$ depends on any element of $M_{r, S}$. Set

$$
B:=\max \left(B_{1}, B_{2} \cdot\left(\mathrm{d}(r)^{\delta_{0}}-\mathrm{n}(r)^{\delta_{0}}\right)\right) .
$$

We argue that $\mathrm{L}(x)$ is an AAP with difference $\mathrm{d}(r)-\mathrm{n}(r)$ and bound $B$. Observe that if $S=\mathbb{N}$ then $B=0$. Now if $c_{j} \geq \mathrm{n}(r)^{s_{m}-s_{j}}$ for some $j \in \llbracket 0, m-1 \rrbracket$ then, by carrying out the transformation $\mathrm{n}(r)^{s_{m}-s_{j}} r^{s_{j}}=\mathrm{d}(r)^{s_{m}-s_{j}} r^{s_{m}}$, we can generate a new factorization $z^{\prime} \in \mathrm{Z}(x)$ such that $\left|z^{\prime}\right|-|z| \leq B$. Also note that, in this case, the term $\mathrm{d}(r)^{s_{m}-s_{j}} r^{s_{m}}$ shows up as a summand in the factorization $z^{\prime}$; consequently, we can generate new factorizations of $x$ by applying, any number of times, the identity $\mathrm{n}(r) r^{\alpha}=\mathrm{d}(r) r^{\alpha+1}$ with $\alpha \geq s_{m}$. Hence

$$
\left\{\left|z^{\prime}\right|+l \cdot(\mathrm{d}(r)-\mathrm{n}(r)) \mid l \in \mathbb{N}\right\} \subseteq \mathrm{L}(x),
$$

and the set $\mathrm{L}(x)$ is an AAP with difference $\mathrm{d}(r)-\mathrm{n}(r)$ and bound $B$. On the other hand, if $c_{j}<\mathrm{n}(r)^{s_{m}-s_{j}}$ for each $j \in \llbracket 0, m-1 \rrbracket$ then consider any factorization $z^{\prime} \in \mathrm{Z}(x)$ satisfying that $\left|z^{\prime}\right|-\left|z_{\min }\right|>B$. By Remark 3.4, there exist factorizations $z_{\min }=$ $z_{1}, \ldots, z_{k}=z^{\prime} \in \mathbf{Z}(x)$ such that, for each $i \in \llbracket 1, k-1 \rrbracket$, we have

$$
\left|z_{i+1}\right|-\left|z_{i}\right|=\mathrm{d}(r)^{\delta_{n}}-\mathrm{n}(r)^{\delta_{n}}
$$

for some $n \in \mathbb{N}$. Since $\left|z^{\prime}\right|-\left|z_{\text {min }}\right|>B_{2} \cdot\left(\mathrm{d}(r)^{\delta_{0}}-\mathrm{n}(r)^{\delta_{0}}\right)$, there exists $j \in \llbracket 1, k-1 \rrbracket$ such that $z_{j+1}$ was obtained from $z_{j}$ by carrying out a transformation of the form $\mathrm{n}(r)^{\delta_{t}} r^{s_{t}}=\mathrm{d}(r)^{\delta_{t}} r^{s_{t+1}}$ for some $t \geq m$. As before, a term $\mathrm{n}(r)^{\delta_{t}} r^{s_{t}}$ shows up in the factorization $z_{j}$, which means that we can generate new factorizations of $x$ by applying 
the identity $\mathrm{n}(r) r^{\alpha}=\mathrm{d}(r) r^{\alpha+1}$ with $\alpha \geq s_{m}$ an arbitrary number of times. Since $\mathrm{d}(r)-\mathrm{n}(r) \mid \mathrm{d}(r)^{\delta_{n}}-\mathrm{n}(r)^{\delta_{n}}$ for every $n \in \mathbb{N}$, we have that

$$
\left|z^{\prime}\right| \in\left\{\left|z_{j}\right|+l \cdot(\mathrm{d}(r)-\mathrm{n}(r)) \mid l \in \mathbb{N}\right\} \subseteq \mathrm{L}(x) .
$$

Observe that $\left|z_{j}\right|<\left|z^{\prime}\right|$. If $\left|z_{j}\right|-\left|z_{\min }\right|>B$ then, by the same token, there exists $z_{t} \in \mathrm{Z}(x)$ such that

$$
\left|z_{j}\right| \in\left\{\left|z_{t}\right|+l \cdot(\mathrm{d}(r)-\mathrm{n}(r)) \mid l \in \mathbb{N}\right\} \subseteq \mathrm{L}(x)
$$

and $\left|z_{t}\right|<\left|z_{j}\right|$. Our argument follows inductively.

CASE 2: $r>1$. Let us denote by $B_{2}$ the maximum number of times we can consecutively apply the identity $\mathrm{d}(r)^{\delta_{\alpha-1}} r^{s_{\alpha}}=\mathrm{n}(r)^{\delta_{\alpha-1}} r^{s_{\alpha-1}}$ with $\alpha \in \llbracket 1, m \rrbracket$ to increase the length of the prefix of a factorization $z=\sum_{i=0}^{k} d_{i} r^{s_{i}}$ satisfying that $d_{i}<\mathrm{n}(r)^{\delta_{i}}$ for each $i \in \llbracket 1, m \rrbracket$. The number $B_{2}$ does not depend on any element $x \in M_{r, S}$. Notice that if $S=\mathbb{N}$ then $B_{2}=0$. Consider the factorization $z_{1}=z^{\prime}+\sum_{i=m+1}^{n} c_{i} r^{s_{i}} \in \mathrm{Z}(x)$, where $z^{\prime}$ is the factorization of maximum length of $\pi\left(\sum_{i=0}^{m} c_{i} r^{s_{i}}\right)$. It is not hard to see that the inequality $\left|z_{1}\right|-\left|z_{\min }\right| \leq B_{2} \cdot\left(\mathrm{n}(r)^{\delta_{0}}-\mathrm{d}(r)^{\delta_{0}}\right)$ holds. By [35, Theorem 5.6], the monoid $M_{r, S}$ is an FFM, so there is no loss in assuming that no atom bigger than $r^{s_{n}}$ divides $x$ in $M_{r, S}$.

Next we describe a procedure to generate, iteratively, overlapping arithmetic sequences of lengths of $x$ with difference $\mathrm{n}(r)-\mathrm{d}(r)$. Set $z_{1}^{*}:=z_{1}$, and let us denote by $K_{1}$ the maximum number of times we can consecutively apply the identity $\mathrm{d}(r)^{\delta_{\alpha-1}} r^{s_{\alpha}}=\mathrm{n}(r)^{\delta_{\alpha-1}} r^{s_{\alpha-1}}$ with $\alpha \in \llbracket m+1, n \rrbracket$ to increase the length of $z_{1}^{*}$. Additionally, set

$$
\sigma_{1}:=\left\{\left|z_{1}^{*}\right|+l \cdot(\mathrm{n}(r)-\mathrm{d}(r)) \mid l \in \llbracket 0, K_{1} \rrbracket\right\},
$$

and note that $\sigma_{1} \subseteq \mathrm{L}(x)$. Suppose that, for some $j \in \mathbb{N}^{\bullet}$, we already defined $\sigma_{j}, K_{j}$, and $z_{j}^{*}=\sum_{i=0}^{n} c_{j, i} r^{s_{i}} \in \mathbf{Z}(x)$, where $c_{j, i}<\mathrm{d}(r)^{\delta_{i-1}}$ for each $i \in \llbracket 1, m \rrbracket$, such that

$$
\sigma_{j}=\left\{\left|z_{j}^{*}\right|+l \cdot(\mathrm{n}(r)-\mathrm{d}(r)) \mid l \in \llbracket 0, K_{j} \rrbracket\right\} \subseteq \mathrm{L}(x) .
$$

By hypothesis of induction, the nonnegative integer $K_{j}$ represents the maximum number of times we can consecutively apply the identity $\mathrm{d}(r)^{\delta_{\alpha-1}} r^{s_{\alpha}}=\mathrm{n}(r)^{\delta_{\alpha-1}} r^{s_{\alpha-1}}$ with $\alpha \in \llbracket m+1, n \rrbracket$ to increase the length of $z_{j}^{*}$. Now if $z_{j}^{*}$ is the factorization of maximum length of $x$ (i.e., $K_{j}=0$ ) then our procedure stops at this factorization. Otherwise, there exists $u \in \llbracket m+1, n \rrbracket$ such that $c_{j, u} \geq \mathrm{d}(r)^{\delta_{u-1}}=\mathrm{d}(r)$. After consecutively applying the transformations

$$
\mathrm{d}(r) r^{s_{u}}=\mathrm{n}(r) r^{s_{u-1}}, \ldots, \mathrm{d}(r) r^{s_{m+1}}=\mathrm{n}(r) r^{s_{m}}
$$

to increase the length of $z_{j}^{*}$, we obtain a factorization $z_{j, j+1}=\sum_{i=0}^{n} d_{j+1, i} r^{s_{i}} \in \mathrm{Z}(x)$, where $d_{j+1, i}<\mathrm{d}(r)^{\delta_{i-1}}$ for each $i \in \llbracket 1, m-1 \rrbracket$ and $d_{j+1, m}<\mathrm{d}(r)^{\delta_{m-1}}+\mathrm{n}(r)$. Clearly, the inequalities

$$
\left|z_{j}^{*}\right|<\left|z_{j, j+1}\right| \leq\left|z_{j}^{*}\right|+K_{j} \cdot(\mathrm{n}(r)-\mathrm{d}(r))
$$


hold. Consider the factorization $z_{j+1}^{*}=z^{\prime \prime}+\sum_{i=m+1}^{n} d_{j+1, i} r^{s_{i}} \in \mathrm{Z}(x)$, where $z^{\prime \prime}$ is the factorization of maximum length of $\pi\left(\sum_{i=0}^{m} d_{j+1, i} r^{s_{i}}\right)$. Note that if $S=\mathbb{N}$ then $z_{j+1}^{*}=$ $z_{j, j+1}$. It is easy to see that $\left|z_{j, j+1}\right| \leq\left|z_{j+1}^{*}\right|$, and rewriting $z_{j+1}^{*}$ as $z_{j+1}^{*}=\sum_{i=0}^{n} c_{j+1, i} r^{s_{i}}$ we have $c_{j+1, i}<\mathrm{d}(r)^{\delta_{i-1}}$ for each $i \in \llbracket 1, m \rrbracket$. Let us denote by $K_{j+1}$ the maximum number of times we can consecutively apply the identity $\mathrm{d}(r)^{\delta_{\alpha-1}} r^{s_{\alpha}}=\mathrm{n}(r)^{\delta_{\alpha-1}} r^{s_{\alpha-1}}$ with $\alpha \in \llbracket m+1, n \rrbracket$ to increase the length of $z_{j+1}^{*}$. Then set

$$
\sigma_{j+1}:=\left\{\left|z_{j+1}^{*}\right|+l \cdot(\mathrm{n}(r)-\mathrm{d}(r)) \mid l \in \llbracket 0, K_{j+1} \rrbracket\right\} \subseteq \mathrm{L}(x) .
$$

If $K_{j+1} \cdot(\mathrm{n}(r)-\mathrm{d}(r)) \geq\left|z_{j+1}^{*}\right|-\left|z_{j, j+1}\right|$ then we have that

$$
\left|z_{j+1}^{*}\right| \in \sigma_{(j, j+1)}:=\left\{\left|z_{j, j+1}\right|+l \cdot(\mathrm{n}(r)-\mathrm{d}(r)) \mid l \in \llbracket 0, K_{j+1} \rrbracket\right\} \subseteq \mathrm{L}(x),
$$

where the inclusion holds because the suffixes of $z_{j, j+1}$ and $z_{j+1}^{*}$ coincide. This means that we have three overlapping arithmetic sequences $\sigma_{j}, \sigma_{(j, j+1)}$, and $\sigma_{j+1}$ of lengths of $x$ with difference $\mathrm{n}(r)-\mathrm{d}(r)$. Combining these sequences we obtain a new arithmetic sequence $\sigma^{*}$ starting at $\left|z_{j}^{*}\right|$, containing $\left|z_{j+1}^{*}\right|$, and with difference $\mathrm{n}(r)-\mathrm{d}(r)$. Now update $\sigma_{j}$ as $\sigma^{*}$. On the other hand, if

$$
K_{j+1} \cdot(\mathrm{n}(r)-\mathrm{d}(r))<\left|z_{j+1}^{*}\right|-\left|z_{j, j+1}\right|
$$

then our procedure stops at the factorization $z_{j+1}^{*}$. Notice that our algorithm eventually stops. In fact, in each step we generate from $z_{j}^{*}$ a new factorization $z_{j, j+1}$ such that $\left|z_{j}^{*}\right|<\left|z_{j, j+1}\right|$; consequently, the procedure stops after finitely many iterations by [35, Proposition 4.5].

The algorithm that we just described yields a sequence of nonnegative integers $K_{1}, \ldots, K_{k+1}$, a sequence of factorizations $z_{1}^{*}, \ldots, z_{k+1}^{*} \in \mathbf{Z}(x)$, and a sequence of finite arithmetic progressions $\sigma_{1}, \ldots, \sigma_{k+1}$ with difference $\mathrm{n}(r)-\mathrm{d}(r)$ such that $\sigma_{i}$ starts at $\left|z_{i}^{*}\right|$ for each $i \in \llbracket 1, k+1 \rrbracket$ and contains $\left|z_{i+1}^{*}\right|$ for each $i \in \llbracket 1, k-1 \rrbracket$. Moreover, we have $\sigma_{i} \subseteq \mathrm{L}(x)$ for each $i \in \llbracket 1, k+1 \rrbracket$. We already showed that $\left|z_{1}^{*}\right|-\left|z_{\min }\right| \leq B_{2} \cdot\left(\mathrm{n}(r)^{\delta_{0}}-\mathrm{d}(r)^{\delta_{0}}\right)$, where $B_{2}$ does not depend on $x$ and $z_{\text {min }}$ is the factorization of minimum length of $x$. Now let us suppose that the algorithm stopped at the factorization $z_{k+1}^{*}$. Note that if $S=\mathbb{N}$ then $z_{k+1}^{*}$ is the factorization of maximum length of $x$, which implies that $\mathrm{L}(x)$ is an arithmetic sequence with difference $\mathrm{n}(r)-\mathrm{d}(r)$. Since our procedure stopped at $z_{k+1}^{*}$, it is not hard to see that

$$
K_{k+1} \leq\left(\left|z_{k+1}^{*}\right|-\left|z_{k, k+1}\right|\right)(\mathrm{n}(r)-\mathrm{d}(r))^{-1},
$$

where $z_{k, k+1}=\sum_{i=0}^{n} d_{i} r^{s_{i}} \in \mathbf{Z}(x), z_{k+1}^{*}=z^{\prime \prime}+\sum_{i=m+1}^{n} d_{i} r^{s_{i}} \in \mathbf{Z}(x)$, and $z^{\prime \prime}$ is the factorization of maximum length of $\pi\left(\sum_{i=0}^{m} d_{i} r^{s_{i}}\right)$ with $d_{i}<\mathrm{d}(r)^{\delta_{i-1}}$ for each $i \in \llbracket 1, m-$ 1】 and $d_{m}<\mathrm{d}(r)^{\delta_{m-1}}+\mathrm{n}(r)$. Consequently, there exists $B_{3} \in \mathbb{N}$ (which does not depend on $x$ ) such that $\left|z_{k+1}^{*}\right|-\left|z_{k, k+1}\right| \leq B_{3}$. Let $z_{\beta}=\sum_{i=0}^{n} e_{i} r^{s_{i}} \in \mathrm{Z}(x)$ be the factorization we obtain after consecutively applying the identity $\mathrm{d}(r)^{\delta_{\alpha-1}} r^{s_{\alpha}}=\mathrm{n}(r)^{\delta_{\alpha-1}} r^{s_{\alpha-1}}$ with $\alpha \in \llbracket m+1, n \rrbracket$ as many times as we can (i.e., $\left.K_{k+1}\right)$ to increase the length of $z_{k+1}^{*}$. 
Evidently, the inequalities

$$
\left|z_{\beta}\right|-\left|z_{k+1}^{*}\right| \leq K_{k+1} \cdot(\mathrm{n}(r)-\mathrm{d}(r)) \leq B_{3}
$$

hold. Moreover, we have that $e_{i}<\mathrm{d}(r)^{\delta_{i-1}}$ for every $i \in \llbracket 1, n \rrbracket$ with $i \neq m$ and

$$
e_{m}<\mathrm{d}(r)^{\delta_{m-1}}+\mathrm{n}(r) \cdot K_{k+1} \leq \mathrm{d}(r)^{\delta_{m-1}}+\mathrm{n}(r) \cdot B_{3} \cdot(\mathrm{n}(r)-\mathrm{d}(r))^{-1} .
$$

It is easy to see that there exists $B_{4} \in \mathbb{N}$ (which does not depend on $x$ either) such that $\left|z_{\max }\right|-\left|z_{\beta}\right| \leq B_{4}$, where $z_{\max } \in \mathbf{Z}(x)$ is the factorization of maximum length of $x$. Hence $\left|z_{\max }\right|-\left|z_{k, k+1}\right| \leq 2 B_{3}+B_{4}$. Set

$$
B:=\max \left(B_{2} \cdot\left(\mathrm{n}(r)^{\delta_{0}}-\mathrm{d}(r)^{\delta_{0}}\right), 2 B_{3}+B_{4}\right) .
$$

Since $\left|z_{k, k+1}\right| \in \sigma_{k}$, we can conclude that the set $\mathrm{L}(x)$ is an AAP with difference $\mathrm{n}(r)-\mathrm{d}(r)$ and bound $B$ for all $x \in M_{r, S}$.

Corollary 4.2. [12, Theorem 3.3] Let $M_{r, \mathbb{N}}$ be a nontrivial atomic rational cyclic semiring. Then $\mathrm{L}(x)$ is an arithmetic progression with difference $|\mathrm{n}(r)-\mathrm{d}(r)|$ for all $x \in M_{r, \mathbb{N}}$.

Since the sets of lengths of atomic rational cyclic semirings are arithmetic sequences, it is natural to wonder whether there exist other atomic exponential Puiseux semirings whose sets of lengths are also arithmetic sequences. We now answer this question negatively.

Corollary 4.3. Let $M_{r, S}$ be a nontrivial atomic exponential Puiseux semiring. Then $\left|\Delta\left(M_{r, S}\right)\right|=1$ if and only if $S=\mathbb{N}$.

Proof. The reverse implication follows from Corollary 3.11. As for the remaining implication, if $\left|\Delta\left(M_{r, S}\right)\right|=1$ then, by Proposition 3.10, we have $\delta_{i}=\delta_{j}$ for all $i, j \in \mathbb{N}$, which implies $S=\mathbb{N}$.

Remark 4.4. A monoid $M$, which is not necessarily reduced, is strongly primary if $M \neq M^{\times}$and for every $x \in M \backslash M^{\times}$there exists $n \in \mathbb{N}$ such that $\left(M \backslash M^{\times}\right)^{n} \subseteq x M$. Strongly primary monoids play a central role in factorization theory, and they have been widely investigated (see, for instance, $[24,30]$ ). It is known that sets of lengths of certain strongly primary monoids are AAPs with a common bound $B$ ( [26, Theorem 4.3.6]). However, nontrivial exponential Puiseux semirings are not strongly primary. In fact, let $M_{r, S}$ be a nontrivial atomic exponential Puiseux semiring, and fix an atom $r^{k} \in \mathcal{A}\left(M_{r, S}\right)$ with $k \in S$. Observe that, for any $n \in \mathbb{N} \bullet$, we have that $r^{s_{k}} \nmid_{M_{r, S}} \sum_{i=1}^{n} r^{s_{k+i}}$ by Lemma 3.2 and Lemma 3.3. Therefore, the monoid $M_{r, S}$ is not strongly primary.

The bounds provided in Theorem 4.1 are not tight, and the next example sheds some light upon this observation. 
Example 4.5. Let $r=3 / 4$ and $S=\{0,5,7\} \cup \mathbb{N}_{\geq 8}$. By Theorem 4.1, every $L \in$ $\mathcal{L}\left(M_{r, S}\right)$ is an AAP with difference 1 and bound $B=717739$. Indeed, with notation as in CASE 1 of Theorem 4.1, we have $B_{1}=4^{7}-3^{7}$. On the other hand, $B_{2}$ is equal to the number of times we can apply the identity $\mathrm{n}(r)^{\delta_{\alpha}} r^{s_{\alpha}}=\mathrm{d}(r)^{\delta_{\alpha}} r^{s_{\alpha+1}}$ with $\alpha \in\{0,1\}$ to increase the length of the factorization $z=\left(3^{7}-1\right)+\left(3^{2}-1\right)(3 / 4)^{5}$. Consequently,

$$
B_{2}=\left\lfloor\frac{\left(3^{7}-1\right)}{3^{5}}\right\rfloor+\left\lfloor\frac{4^{5}\left\lfloor\frac{\left(3^{7}-1\right)}{3^{5}}\right\rfloor+3^{2}-1}{9}\right\rfloor=919 .
$$

Thus, $B=717739$. However, it is not hard to see that every $L \in \mathcal{L}\left(M_{r, S}\right)$ is an AAP with difference 1 and bound $B^{\prime}=\left(4^{5}-3^{5}\right)+\left(4^{2}-3^{2}\right)=788<717739$.

Motivated by Example 4.5, we pose the following question.

Question 4.6. Given an atomic exponential Puiseux semiring $M_{r, S}$, what is the smallest nonnegative integer $B$ for which $M_{r, S}$ is an $A A P$ with difference $|\mathrm{n}(r)-\mathrm{d}(r)|$ and bound $B$ ?

We now present unions of sets of lengths, a factorization invariant introduced by Chapman and Smith [14]. For a positive integer $k$, denote by $\mathcal{U}_{k}(M)$ the set of positive integers $m$ for which there exist $a_{1}, \ldots, a_{k}, a_{1}^{\prime}, \ldots, a_{m}^{\prime} \in \mathcal{A}(M)$ such that

$$
a_{1}+\cdots+a_{k}=a_{1}^{\prime}+\cdots+a_{m}^{\prime} .
$$

It is said that $\mathcal{U}_{k}(M)$ is the union of sets of lengths of $M$ containing $k$.

Next we show that, as it is the case for atomic rational cyclic semirings, unions of sets of lengths of nontrivial atomic exponential Puiseux semirings are arithmetic sequences with difference $|\mathrm{n}(r)-\mathrm{d}(r)|$.

Proposition 4.7. Let $M_{r, S}$ be a nontrivial atomic exponential Puiseux semiring. For every $k \in \mathbb{N}^{\bullet}$, the set $\mathcal{U}_{k}\left(M_{r, S}\right)$ is an arithmetic sequence with difference $|\mathrm{n}(r)-\mathrm{d}(r)|$.

Proof. Assume that $r<1$. Fix $k \in \mathbb{N} \bullet$, and let $x \in M_{r, S}$ such that $k \in \mathrm{L}(x)$. Let $m \in \mathrm{L}(x)$. In addition, let $z_{\min } \in \mathrm{Z}(x)$ be the factorization of minimum length of $x$, and let $z, z^{\prime \prime} \in \mathrm{Z}(x)$ be factorizations of lengths $m$ and $k$, respectively. By Remark 3.4, there exist factorizations $z_{\min }=z_{1}, \ldots, z_{n}=z \in \mathbf{Z}(x)$ such that, for each $i \in \llbracket 1, n-1 \rrbracket$, we have $\left|z_{i+1}\right|-\left|z_{i}\right|=\mathrm{d}(r)^{\delta_{\alpha}}-\mathrm{n}(r)^{\delta_{\alpha}}$ for some $\alpha \in \mathbb{N}$. Now consider the element $y=r^{F(S)+1} \cdot x \in M_{r, S}$ and, for each $i \in \llbracket 1, n \rrbracket$, let $z_{i}^{\prime}=r^{F(S)+1} \cdot z_{i} \in \mathbf{Z}(y)$. Clearly, $\left|z_{i}^{\prime}\right|=\left|z_{i}\right|$ for every $i \in \llbracket 1, n \rrbracket$. Take $j \in \llbracket 1, n-1 \rrbracket$, and notice that if $z_{j}=\sum_{l=0}^{u} c_{l} r^{s_{l}}$ with coefficients $c_{0}, \ldots, c_{u} \in \mathbb{N}$ then $z_{j+1}$ was obtained from $z_{j}$ by carrying out the transformation $\mathrm{n}(r)^{\delta_{t}} r^{s_{t}}=\mathrm{d}(r)^{\delta_{t}} r^{s_{t+1}}$ for some $t \in \llbracket 0, u \rrbracket$. This implies that we can obtain the factorization $z_{j+1}^{\prime}$ from $z_{j}^{\prime}=\sum_{l=0}^{u} c_{l} r^{s_{l}+F(S)+1}$ by applying the transformation

$$
\mathrm{n}(r)^{\delta_{t}} r^{s_{t}+F(S)+1}=\mathrm{d}(r)^{\delta_{t}} r^{s_{t+1}+F(S)+1},
$$


which is valid in this context; in fact, carrying out this transformation once is equivalent to applying $\left(\mathrm{d}(r)^{\delta_{t}}-\mathrm{n}(r)^{\delta_{t}}\right)(\mathrm{d}(r)-\mathrm{n}(r))^{-1}$ times the identity $\mathrm{n}(r) r^{n}=\mathrm{d}(r) r^{n+1}$ with $n>F(S)$. In other words, there exist factorizations $z_{j}^{\prime}=z_{1}^{*}, \ldots, z_{s}^{*}=z_{j+1}^{\prime} \in \mathrm{Z}(y)$ such that $\left|z_{i+1}^{*}\right|-\left|z_{i}^{*}\right|=\mathrm{d}(r)-\mathrm{n}(r)$ for each $i \in \llbracket 1, s-1 \rrbracket$. Hence $\mathrm{L}(y)$ contains a finite arithmetic sequence starting at $\left|z_{1}^{\prime}\right|$, ending at $\left|z_{n}^{\prime}\right|=m$, and with difference $\mathrm{d}(r)-\mathrm{n}(r)$. Similarly, $\mathrm{L}(y)$ contains a finite arithmetic sequence starting at $\left|z_{1}^{\prime}\right|$, ending at $\left|z^{\prime \prime}\right|=k$, and with difference $\mathrm{d}(r)-\mathrm{n}(r)$. Since both arithmetic sequences have an element in common, namely $\left|z_{1}^{\prime}\right|$, we can think of $\mathcal{U}_{k}\left(M_{r, S}\right)$ as the union of infinitely many finite arithmetic sequences with difference $\mathrm{d}(r)-\mathrm{n}(r)$ containing $k$, from which our result follows.

We can use the same idea to tackle the case where $r \in \mathbb{Q}_{>1} \backslash \mathbb{N}$. We leave the details to the reader.

Geroldinger and Schmid [31] investigated the intersection of systems of sets of lengths of numerical monoids. In particular, they proved $\cap \mathcal{L}(M)=\{\{0\},\{1\},\{2\}\}$, where the intersection is taken over all numerical monoids $M \neq \mathbb{N}$. Gotti [36, Corollary 5.7] showed that if we take the previous intersection over all nontrivial atomic Puiseux monoids then we obtain $\cap \mathcal{L}(M)=\{\{0\},\{1\}\}$. We can now describe the intersection of systems of sets of lengths of nontrivial atomic exponential Puiseux semirings $M_{r, S}$ with $|\mathrm{n}(r)-\mathrm{d}(r)|$ fixed.

Proposition 4.8. For every $m \in \mathbb{N}^{\bullet}$, we have

$$
\bigcap \mathcal{L}\left(M_{r, S}\right)=\{\{n\} \mid n \in \mathbb{N}\},
$$

where the intersection is taken over all nontrivial atomic exponential Puiseux semirings with $|\mathrm{n}(r)-\mathrm{d}(r)|=m$.

Proof. Fix $m \in \mathbb{N} \bullet$. Let $M_{r, S}$ be a nontrivial atomic exponential Puiseux semiring with $|\mathrm{n}(r)-\mathrm{d}(r)|=m$, and let $n$ be a nonnegative integer. If $n=0$ then we have that $\{0\} \in \mathcal{L}\left(M_{r, S}\right)$ since $\mathrm{L}(0)=\{0\}$, so we may assume that $n>0$. Now consider the factorization $z_{n}=\sum_{i=1}^{n} r^{s_{i}}$, where $s_{i} \in S$ for each $i \in \llbracket 1, n \rrbracket$. By lemmas 3.2 and 3.3, we have $\mathrm{Z}\left(\pi\left(z_{n}\right)\right)=\left\{z_{n}\right\}$ which, in turn, implies that $\{n\} \in \mathcal{L}\left(M_{r, S}\right)$. Hence the inclusion $\{\{n\} \mid n \in \mathbb{N}\} \subseteq \cap \mathcal{L}\left(M_{r, S}\right)$ holds.

Let $p$ be a prime number satisfying that $p>m$, and take $r=(p+m) / p$. For a numerical monoid $S$, note that $M_{r, S}$ is an FFM by [35, Theorem 5.6]. Consequently, all sets of lengths in $\cap \mathcal{L}\left(M_{r, S}\right)$, where the intersection is taken over all nontrivial atomic exponential Puiseux semirings with $|\mathrm{n}(r)-\mathrm{d}(r)|=m$, have finite cardinality. Now consider the rational cyclic semiring $M_{q, \mathbb{N}}$ with $q=r^{-1}$. By [1, Proposition 3.7], the monoid $M_{q, \mathbb{N}}$ is atomic. Moreover, for each $x \in M_{q, \mathbb{N}}$, we have $|\mathrm{L}(x)| \in\{1, \infty\}$. In fact, if $z=\sum_{i=0}^{n} c_{i} q^{s_{i}} \in \mathrm{Z}(x)$ is not the factorization of maximum length of $x$ then $c_{j} \geq \mathrm{n}(q)$ for some $j \in \llbracket 0, n \rrbracket$ by Lemma 3.2. By performing the transformation $\mathrm{n}(q) q^{s_{j}}=\mathrm{d}(q) q^{s_{j}+1}$, we obtain a factorization $z_{1} \in \mathrm{Z}(x)$ such that $|z|<\left|z_{1}\right|$. Since $z_{1}$ is not the factorization of maximum length of $x$ either, we can repeat this reasoning 
H. POLO

to obtain $z_{2} \in \mathrm{Z}(x)$, which is not the factorization of maximum length of $x$, such that $\left|z_{1}\right|<\left|z_{2}\right|$, and so on. Consequently, we have $|\mathrm{L}(x)|=\infty$, which concludes our argument.

\section{Omega Primalities and Tame Degrees}

Here we analyze the omega primality in the context of nontrivial atomic exponential Puiseux semirings, but first let us introduce some definitions.

Definition 5.1. Let $M$ be an atomic monoid, and let $x$ be a nonzero element of $M$.

(1) Let $\omega(x)$ denote the smallest $n \in \overline{\mathbb{N}}$ satisfying that if $\left.x\right|_{M} a_{1}+\cdots+a_{m}$ for some $a_{1}, \ldots, a_{m} \in \mathcal{A}(M)$ then $\left.x\right|_{M} a_{i_{1}}+\cdots+a_{i_{k}}$, where $\left\{i_{1}, \ldots, i_{k}\right\} \subseteq \llbracket 1, m \rrbracket$ and $k \leq n$. In addition, set

$$
\omega(M):=\sup \{\omega(a) \mid a \in \mathcal{A}(M)\} .
$$

The elements $\omega(x)$ and $\omega(M)$ are called the omega primalities of $x$ and $M$, respectively.

(2) For $a \in \mathcal{A}(M)$, let $\mathrm{t}(a)$ denote the smallest $n \in \overline{\mathbb{N}}$ satisfying that if $\mathrm{Z}(y) \cap(a+$ $\mathbf{Z}(M)) \neq \emptyset$ for some $y \in M$ and $z \in \mathbf{Z}(y)$ then there exists $z^{\prime} \in \mathbf{Z}(y) \cap(a+\mathbf{Z}(M))$ such that $\mathrm{d}\left(z, z^{\prime}\right) \leq n$. In addition, set

$$
\mathrm{t}(M):=\sup \{\mathrm{t}(a) \mid a \in \mathcal{A}(M)\} .
$$

The elements $\mathrm{t}(a)$ and $\mathrm{t}(M)$ are called the tame degrees of $a$ and $M$, respectively.

With notation as in Definition 5.1, the omega function $\omega: M \rightarrow \overline{\mathbb{N}}$ was introduced by Geroldinger [22], and it measures how far is a nonzero element from being prime. Note that $x \in M^{\bullet}$ is prime if and only if $\omega(x)=1$. Numerous papers have been dedicated to study the computational aspects of this factorization invariant (e.g., [3, 19]).

Given a nontrivial atomic exponential Puiseux semiring $M_{r, S}$, we have $\rho\left(M_{r, S}\right)=\infty$ by [38, Theorem 3.2], which implies $\omega\left(M_{r, S}\right)=\infty$ and $\mathrm{t}\left(M_{r, S}\right)=\infty$ by [28, Proposition 3.6 and Proposition 3.5]. Next we offer several characterizations of nontrivial atomic exponential Puiseux semirings with finite omega functions, but first we collect some technical results and introduce an additional definition.

Lemma 5.2. Let $M_{r, S}$ be a nontrivial atomic exponential Puiseux semiring with $r>1$. If $x \in M_{r, S}$ and $k \in \mathbb{N}$ such that $\left.r^{s_{k}}\right|_{M_{r, S}} x$ then, for every $z=\sum_{i=0}^{n} c_{i} r^{s_{i}} \in \mathrm{Z}(x)$ with coefficients $c_{0}, \ldots, c_{n} \in \mathbb{N}$, we have that either

$$
\left.r^{s_{k}}\right|_{M_{r, S}} \pi\left(\sum_{i=0}^{k} c_{i} r^{s_{i}}\right) \quad \text { or }\left.\quad r^{s_{k}}\right|_{M_{r, S}} \pi\left(\sum_{i=k+1}^{n} c_{i} r^{s_{i}}\right) .
$$


Proof. Let $z^{\prime}=\sum_{i=0}^{m} d_{i} r^{s_{i}} \in \mathrm{Z}(x)$ with coefficients $d_{0}, \ldots, d_{m} \in \mathbb{N}$ such that $m \geq k$ and $d_{k} \neq 0$. Clearly, such a factorization $z^{\prime}$ exists given that $\left.r^{s_{k}}\right|_{M_{r, S}} x$. By Remark 3.4, there exist factorizations

$$
z^{\prime}=z_{1}, \ldots, z_{l}=z_{\max }=\sum_{i=0}^{u} e_{i} r^{s_{i}} \in \mathbf{Z}(x)
$$

such that $z_{\max }$ is the factorization of maximum length of $x$ and, for each $i \in \llbracket 1, l-1 \rrbracket$, we have $\left|z_{i+1}\right|-\left|z_{i}\right|=\left|\mathrm{n}(r)^{\delta_{t}}-\mathrm{d}(r)^{\delta_{t}}\right|$ for some $t \in \mathbb{N}$. Since $d_{k} \neq 0$, it is not hard to see that $\left.r^{s_{k}}\right|_{M_{r, S}} \pi\left(\sum_{i=0}^{k} e_{i} r^{s_{i}}\right)$. Now assume that $r^{s_{k}} \nmid_{M_{r, S}} \pi\left(\sum_{i=k+1}^{n} c_{i} r^{s_{i}}\right)$, and let $z^{\prime \prime}$ be the factorization of maximum length of $\pi\left(\sum_{i=k+1}^{n} c_{i} r^{s_{i}}\right)$. Observe that if an atom $r^{s_{t}}$ shows up in the factorization $z^{\prime \prime}$ then $t>k$; otherwise, we can repeatedly apply the identity $\mathrm{d}(r)^{\delta_{\alpha}} r^{s_{\alpha+1}}=\mathrm{n}(r)^{\delta_{\alpha}} r^{s_{\alpha}}$ to transform the factorization $\sum_{i=k+1}^{n} c_{i} r^{s_{i}}$ into $z^{\prime \prime}$ as described in Remark 3.4, but since $z^{\prime \prime}$ would contain atoms smaller than $r^{s_{k+1}}$, at some point we would have to apply the transformation $\mathrm{d}(r)^{\delta_{k}} r^{s_{k+1}}=\mathrm{n}(r)^{\delta_{k}} r^{s_{k}}$, a contradiction. Consequently, we have

$$
\pi\left(\sum_{i=0}^{k} c_{i} r^{s_{i}}\right)=\pi\left(\sum_{i=0}^{k} e_{i} r^{s_{i}}\right)
$$

from which our argument follows.

Lemma 5.3. Let $M_{r, S}$ be a nontrivial atomic exponential Puiseux semiring with $r>1$. Then $\omega\left(r^{s}\right)<\infty$ for $s>F(S)$.

Proof. Let $m \in \mathbb{N}$ such that $s_{m} \geq F(S)+1$, and set $K:=\max \left(\mathrm{d}(r), \sum_{i=0}^{m-1} \mathrm{n}(r)^{s_{m}-s_{i}}\right)$. We shall prove that $\omega\left(r^{s_{m}}\right) \leq K$. Let $x \in M_{r, S}$ such that $\left.r^{s_{m}}\right|_{M_{r, S}} x$, and consider a factorization $z=\sum_{i=0}^{n} c_{i} r^{s_{i}} \in \mathrm{Z}(x)$ with coefficients $c_{0}, \ldots, c_{n} \in \mathbb{N}$. There is no loss in assuming that $m<n$ and $c_{m}=0$. By Lemma 5.2, we have that either

$$
\left.r^{s_{m}}\right|_{M_{r, S}} \pi\left(\sum_{i=0}^{m} c_{i} r^{s_{i}}\right) \quad \text { or }\left.\quad r^{s_{m}}\right|_{M_{r, S}} \pi\left(\sum_{i=m+1}^{n} c_{i} r^{s_{i}}\right) .
$$

In the latter case, there exists $j \in \llbracket m+1, n \rrbracket$ such that $c_{j} \geq \mathrm{d}(r)$ by Lemma 3.3 and, obviously, $\left.r^{s_{m}}\right|_{M_{r, S}} \mathrm{~d}(r) r^{s_{j}}$. In other words, $r^{s_{m}}$ divides a sub-factorization of $z$ with length at most $K$. Now suppose that $\left.r^{s_{m}}\right|_{M_{r, S}} \pi\left(\sum_{i=0}^{m} c_{i} r^{s_{i}}\right)$. Without loss of generality, we can assume that $K<\sum_{i=0}^{m-1} c_{i}$, so $m \neq 0$. Consequently, there exists $j \in \llbracket 0, m-1 \rrbracket$ such that $c_{j} \geq \mathrm{n}(r)^{s_{m}-s_{j}}$, but $\left.r^{s_{m}}\right|_{M_{r, S}} \mathrm{n}(r)^{s_{m}-s_{j}} r^{s_{j}}$. Therefore, the inequalities $\omega\left(r^{s_{m}}\right) \leq K<\infty$ hold.

Definition 5.4. Let $M$ be an atomic monoid and $\sigma$ a binary relation on $\mathrm{Z}(M)$.

(1) Let $G_{\sigma}$ be the graph whose vertices are the $\mathcal{R}$-classes of $\mathbf{Z}(M)$ and whose edges are the pairs $\left(R, R^{\prime}\right)$ satisfying that $R \neq R^{\prime}$ and $\left(z, z^{\prime}\right) \in \sigma \cup \sigma^{-1}$ for some $z \in R$ and $z^{\prime} \in R^{\prime}$. 
(2) We say that $M$ is $A C C P$-presentable provided that $\sigma^{\prime} \subseteq \sim_{M}$ is a presentation of $M$ if and only if, for every $x \in M$, all the $\mathcal{R}$-classes in $\pi^{-1}(x)$ are in the same connected component of $G_{\sigma^{\prime}}$.

Remark 5.5. Let $M_{r, S}$ be a nontrivial atomic exponential Puiseux semiring, and let $\sigma \subseteq \sim_{M_{r, S}}$. Using Proposition 2.3 and Proposition 3.6, it is easy to prove that if $\sigma$ is a presentation of $M_{r, S}$ then, for every $x \in M_{r, S}$, all the $\mathcal{R}$-classes in $\pi^{-1}(x)$ are in the same connected component of $G_{\sigma}$; we leave the details to the reader. Consequently, proving that $M_{r, S}$ is ACCP-presentable amounts to verify that, for every $\sigma^{\prime} \subseteq \sim_{M_{r, S}}$, if all the $\mathcal{R}$-classes in $\pi^{-1}(x)$ are in the same connected component of $G_{\sigma^{\prime}}$ for every $x \in M_{r, S}$ then $\sigma^{\prime}$ is a presentation of $M_{r, S}$.

It is well known that a monoid satisfying the ACCP is ACCP-presentable ( [7, Theorem 1]). Next we show that these two properties characterize the atomic exponential Puiseux semirings with finite omega functions.

Theorem 5.6. Let $M_{r, S}$ be an atomic exponential Puiseux semiring. The following statements are equivalent.

(1) $r \geq 1$.

(2) $M_{r, S}$ satisfies the $A C C P$.

(3) $M_{r, S}$ is ACCP-presentable.

(4) $\omega(a)<\infty$ for all $a \in \mathcal{A}\left(M_{r, S}\right)$.

Proof. The statements (1) and (2) are equivalent by virtue of [35, Proposition 4.5] and [1, Corollary 4.2], while (4) implies (2) follows from [27, Lemma 3.3].

Now we proceed to argue that (1) implies (4). If $r \in \mathbb{N}$ then this implication trivially holds. Consequently, we may assume that $M_{r, S}$ is nontrivial. Let $m \in \mathbb{N}$ such that $s_{m}=F(S)+1$, and fix $n \in \mathbb{N}$. By Lemma 5.3, the inequality $\omega\left(r^{s_{k}}\right)<\infty$ holds for each $k \geq m$; consequently, we may assume that $n<m$. Then $\left.r^{s_{n}}\right|_{M_{r, S}} \mathrm{~d}(r)^{s_{m}-s_{n}} r^{s_{m}}$ but, for every positive integer $k$, we have that $\omega\left(k \cdot r^{s_{m}}\right) \leq k \cdot \omega\left(r^{s_{m}}\right)<\infty$ by [27, Lemma 3.3] and Lemma 5.3. Applying [27, Lemma 3.3] again, we obtain that $\omega\left(r^{s_{n}}\right)<\infty$, and our argument concludes.

As we mentioned above, a monoid satisfying the ACCP is ACCP-presentable by [7, Theorem 1]. Next we prove that $M_{r, S}$ is not ACCP-presentable when $r<1$. We can write $\mathrm{d}(r)$ as $\mathrm{d}(r)=q \cdot \mathrm{n}(r)+c$ with $q \in \mathbb{N}$ and $1 \leq c<\mathrm{n}(r)$. Since $r<1$, the inequality $q \geq 1$ holds. As before, let $m \in \mathbb{N}$ such that $s_{m}=F(S)+1$. Now let

$$
\rho=\left\{\left(\mathrm{n}(r) r^{n}, \mathrm{~d}(r) r^{n+2}+((q-1) \mathrm{n}(r)+c) r^{n+1}\right) \mid n \in \mathbb{N}_{>F(S)}\right\} \subseteq \mathbf{Z}\left(M_{r, S}\right) \times \mathbf{Z}\left(M_{r, S}\right),
$$

where $F(S)$ represents the Frobenius number of the numerical monoid $S$, and let

$$
\gamma=\left\{\left(\mathrm{n}(r)^{\delta_{n}} r^{s_{n}}, \mathrm{~d}(r)^{\delta_{n}} r^{s_{n+1}}\right) \mid n \in \mathbb{N}_{<m}\right\} \subseteq \mathrm{Z}\left(M_{r, S}\right) \times \mathrm{Z}\left(M_{r, S}\right) .
$$

Take $\sigma=\rho \cup \gamma$. Obviously, the inclusion $\sigma \subseteq \sim_{M_{r, S}}$ holds. Note that, for each $x \in M_{r, S}$, all the $\mathcal{R}$-classes in $\pi^{-1}(x)$ are in the same connected component of $G_{\sigma}$ by 
Proposition 3.6. Let $k \in \mathbb{N}$ such that $k \geq s_{m}$ and $\mathrm{n}(r) r^{k}<r^{s_{n}}$ for each $n \in \llbracket 0, m-1 \rrbracket$. Clearly, if $\left(\mathrm{n}(r) r^{k}, \mathrm{~d}(r) r^{k+1}\right) \in \sigma^{\sharp}$ then $\left(\mathrm{n}(r) r^{k}, \mathrm{~d}(r) r^{k+1}\right) \in \rho^{\sharp}$. However, it is easy to see that if $\left(z, z^{\prime}\right) \in \rho$ then ||$z|-| z^{\prime}||=2 \cdot(\mathrm{d}(r)-\mathrm{n}(r))$, and the same is true for any pair in $\rho^{t}$; consequently, if $\left(z, z^{\prime}\right) \in\left(\rho^{t}\right)^{e}$ then ||$z|-| z^{\prime}||=2 s(\mathrm{~d}(r)-\mathrm{n}(r))$ for some $s \in \mathbb{N}$. Hence $\left(\mathrm{n}(r) r^{k}, \mathrm{~d}(r) r^{k+1}\right) \notin \rho^{\sharp}$. Therefore, $\sigma$ is not a presentation of $M_{r, S}$ which, in turn, implies that $M_{r, S}$ is not ACCP-presentable.

An atomic monoid $M$ is called locally (resp. globally) tame provided that $\mathrm{t}(a)<\infty$ (resp. $\mathrm{t}(M)<\infty)$ for every $a \in \mathcal{A}(M)$. We can now describe the atomic exponential Puiseux semirings that are locally (or globally) tame, but first we need to introduce a definition.

Definition 5.7. Let $M$ be an atomic monoid, and let $x$ be an element of $M$. For $n \in \mathbb{N}^{\bullet}$, we denote by $\mathrm{Z}_{\min }(n, x)$ the set of all factorizations $z \in \mathrm{Z}(M)$ with length at most $n$ satisfying that the smallest sub-factorization of $z$ divisible by $x$ in $M$ is precisely $z$. In addition, set

$$
\tau(x):=\sup _{n} \sup _{z}\left\{\min \mathrm{L}(\pi(z)-x) \mid z \in \mathrm{Z}_{\min }(n, x)\right\} .
$$

Corollary 5.8. Any nontrivial atomic exponential Puiseux monoid is neither locally tame nor globally tame.

Proof. Let $M_{r, S}$ be a nontrivial atomic exponential Puiseux semiring. By virtue of [38, Theorem 3.2], we have $\rho\left(M_{r, S}\right)=\infty$, which implies that $M_{r, S}$ is not globally tame by [26, Theorem 1.6.6]. Now if $r<1$ then $M_{r, S}$ is not locally tame by Theorem 5.6 and [27, Theorem 3.6]. On the other hand, if $r>1$ then it is straightforward to adapt the argument used in the proof of [12, Theorem 5.6] to show that the equality $\tau\left(r^{F(S)+1}\right)=\infty$ holds, so we leave this task to the reader. Our result then follows from [27, Theorem 3.6].

The omega function of a Puiseux monoid satisfying the ACCP is not, in general, a finite function as the following example illustrates.

Example 5.9. Let $M=\left\langle\frac{p-1}{p} \mid p \in \mathbb{P}\right\rangle$. It is easy to see that $\mathcal{A}(M)=\left\{\frac{p-1}{p} \mid\right.$ $p \in \mathbb{P}\}$, which implies that $M$ is atomic. In fact, the monoid $M$ is a BFM by [35, Proposition 4.5]. Let us fix $p \in \mathbb{P}$. A straightforward computation shows that, for $q \in \mathbb{P} \backslash\{p\}$ and $n \in \mathbb{N}$, if $(p-1) /\left.p\right|_{M} n(q-1) / q$ then the inequality $n \geq q$ holds. Consequently, $\omega((p-1) / p) \geq q$ for all $q \in \mathbb{P} \backslash\{p\}$. In other words, we have $\omega((p-1) / p)=$ $\infty$ for each $p \in \mathbb{P}$.

\section{Acknowledgments}

The author wants to thank Felix Gotti and an anonymous referee for valuable feedback that helped improve the quality of this manuscript. While working on the same, the author was generously supported by the CAM Summer Research Fellowship. 


\section{REFERENCES}

[1] S. Albizu-Campos, J. Bringas, and H. Polo: On the atomic structure of exponential Puiseux monoids and semirings, Comm. Algebra 49 (2021) 850-863.

[2] D. D. Anderson, D. F. Anderson, and M. Zafrullah: Factorization in integral domains, J. Pure Appl. Algebra 69 (1990) 1-19.

[3] D. F. Anderson, S. T. Chapman, N. Kaplan, and D. Torkornoo: An algorithm to compute $\omega-$ primality in a numerical monoid, Semigroup Forum 82 (2011) 96-108.

[4] N. R. Baeth, S. T. Chapman, and F. Gotti: Bi-atomic classes of positive semirings, Semigroup Forum (to appear). Preprint on arXiv: https://arxiv.org/pdf/2103.13264.pdf

[5] N. R. Baeth and F. Gotti: Factorizations in upper triangular matrices over information semialgebras, J. Algebra 562 (2020) 466-496.

[6] C. Bowles, S. T. Chapman, N. Kaplan, and D. Reiser: On delta sets of numerical monoids, J. Algebra Appl. 5 (2006) 695-718.

[7] M. Bullejos and P. A. García-Sánchez: Minimal presentations for monoids with the ascending chain condition on principal ideals, Semigroup Forum 85 (2012) 185-190.

[8] P. Cesarz, S. T. Chapman, S. McAdam, and G. J. Schaeffer: Elastic properties of some semirings defined by positive systems, in Commutative Algebra and Its Applications (Eds. M. Fontana, S. E. Kabbaj, B. Olberding, and I. Swanson), Proceedings of the Fifth International Fez Conference on Commutative Algebra and its Applications, Walter de Gruyter, Berlin, 2009, pp. 89-101.

[9] S. T. Chapman, P. A. García-Sánchez, and D. Llena: The catenary and tame degree of numerical monoids, Forum Math. 21 (2009) 117-129.

[10] S. T. Chapman, P. A. García-Sánchez, D. Llena, A. Malyshev, and D. Steinberg: On the delta set and the Betti elements of a BF-monoid, Arab. J. Math. 1 (2012) 53-61.

[11] S. T. Chapman, P. A. García-Sánchez, D. Llena, V. Ponomarenko, and J. C. Rosales: The catenary and tame degree in finitely generated commutative cancellative monoids, Manuscr. Math. 120 (2006) 253-264.

[12] S. T. Chapman, F. Gotti, and M. Gotti: Factorization invariants of Puiseux monoids generated by geometric sequences, Comm. Algebra 48 (2020) 380-396.

[13] S. T. Chapman, F. Gotti, and M. Gotti: When is a Puiseux monoid atomic?, Amer. Math. Monthly 128 (2021) 302-321.

[14] S. T. Chapman and W. W. Smith: Factorization in dedekind domains with finite class group, Israel J. Math. 71 (1990) 65-95.

[15] P. M. Cohn: Bezout rings and their subrings, Proc. Cambridge Phil. Soc. 64 (1968) 251-264.

[16] J. Correa-Morris and F. Gotti: On the additive structure of algebraic valuations cyclic free semirings. Preprint on arXiv: https://arxiv.org/pdf/2008.13073.pdf

[17] J. Coykendall and F. Gotti: On the atomicity of monoid algebras, J. Algebra 539 (2019) 138-151.

[18] W. Gao and A. Geroldinger: On products of $k$ atoms, Monatsh. Math. 156 (2009) 141-157.

[19] J. I. García-García, M. A. Moreno-Frías, and A. Vigneron-Tenorio: Computation of the $\omega$ primality and asymptotic $\omega$-primality with applications to numerical semigroups, Israel J. Math. 206 (2015) 395-411.

[20] P. A. García-Sánchez: An overview of the computational aspects of nonunique factorization invariants, in: Multiplicative Ideal Theory and Factorization Theory, Springer, 2016, pp. 159-181.

[21] P. A. García-Sánchez and J. C. Rosales: Numerical Semigroups, Developments in Mathematics Vol. 20, Springer-Verlag, New York, 2009.

[22] A. Geroldinger: Chains of factorizations in weakly Krull domains, Colloq. Math. 72 (1997) 53-81.

[23] A. Geroldinger: Sets of lengths, Amer. Math. Monthly 123 (2016) 960-988. 
[24] A. Geroldinger, F. Gotti, and S. Tringali: On strongly primary monoids, with a focus on Puiseux monoids, J. Algebra 567 (2021) 310-345.

[25] A. Geroldinger, D. J. Grynkiewicz, and W. A. Schmid: The catenary degree of Krull monoids I, J. Theor. Nombres Bordx. 23 (2011) 137-169.

[26] A. Geroldinger and F. Halter-Koch: Non-unique Factorizations: Algebraic, Combinatorial and Analytic Theory, Pure and Applied Mathematics Vol. 278, Chapman \& Hall/CRC, Boca Raton, 2006.

[27] A. Geroldinger and W. Hassler: Local tameness of v-noetherian monoids, J. Pure Appl. Algebra 212 (2008) 1509-1524.

[28] A. Geroldinger and F. Kainrath: On the arithmetic of tame monoids with applications to Krull monoids and Mori domains, J. Pure Appl. Algebra 214 (2010) 2199-2218.

[29] A. Geroldinger and G. Lettl: Factorization problems in semigroups, Semigroup Forum 40 (1990) $23-38$.

[30] A. Geroldinger and M. Roitman: On strongly primary monoids and domains, Comm. Algebra 48 (2020) 4085-4099.

[31] A. Geroldinger and W. A. Schmid: Which sets are sets of lengths in all numerical monoids?, Banach Center Publications 118 (2019) 181-192.

[32] A. Geroldinger and Q. Zhong: The catenary degree of Krull monoids II, J. Aust. Math. Soc. 98 (2015) 324-354.

[33] A. Geroldinger and Q. Zhong: Sets of arithmetical invariants in transfer Krull monoids, J. Pure Appl. Algebra 223 (2019) 3889-3918.

[34] R. Gilmer: Commutative Semigroup Rings, Chicago Lectures in Mathematics, The University of Chicago Press, London, 1984.

[35] F. Gotti: Increasing positive monoids of ordered fields are FF-monoids, J. Algebra 518 (2019) $40-56$.

[36] F. Gotti: Systems of sets of lengths of Puiseux monoids, J. Pure Appl. Algebra 223 (2019) $1856-1868$.

[37] F. Gotti and M. Gotti: Atomicity and boundedness of monotone Puiseux monoids, Semigroup Forum 96 (2018) 536-552.

[38] F. Gotti and C. O'Neill: The elasticity of Puiseux monoids, J. Commut. Algebra 12 (2020) 319-331.

[39] M. Gotti: On the local k-elasticities of Puiseux monoids, Int. J. Algebra Comput. 29 (2019) $147-158$.

[40] F. Halter-Koch: Finiteness theorem for factorizations, Semigroup Forum 44 (1992) 112-117.

[41] J. M. Howie: An Introduction to Semigroup Theory, Academic Press, London, 1976.

[42] A. Philipp: A characterization of arithmetical invariants by the monoid of relations, Semigroup Forum 81 (2010) 424-434.

[43] H. Polo: On the sets of lengths of Puiseux monoids generated by multiple geometric sequences, Commun. Korean Math. Soc. 35 (2020) 1057-1073.

[44] R. J. Valenza: Elasticity of factorization in number fields, J. Number Theory 36 (1990) 212-218.

[45] A. Zaks: Half-factorial domains, Israel J. Math. 37 (1980) 281-302.

Department of Mathematics, University of Florida, Gainesville, FL 32611, USA

Email address: haroldpolo@ufl.edu 